\title{
On the possibility of grafting conducting polymers into insulating ones
}

\author{
S. Bahçeci ${ }^{a}$, L. Toppare ${ }^{\text {b }}$, E. Yurtsever ${ }^{\mathrm{c}, *}$ \\ ${ }^{a}$ Department of Chemistry, Middle East Technical University, Ankara 06531, Turkey \\ ${ }^{b}$ Department of Chemistry, Bilkent University, Ankara, Turkey \\ 'Department of Chemistry, Kog University, Istanbul, Turkey
}

Received 10 May 1995; revised 29 April 1996; accepted 1 May 1996

\begin{abstract}
The possibility of grafting between conducting polymers, like polypyrrole (PPy) and polyaniline (PAn), and insulating polymers, such as polybisphenol A carbonate (PC) and polyamide (PA), is studied via semi-empirical methods using the AM1 parametrization. Several experimental studies on the issue have previnusly revealed that a chemical interaction exists hetween the couples (PAn-PC, PPy-PC and PPy-PA) during the electrochemical synthesis of PAn and PPy in the insulating host matrices. Here we present additional theoretical evidence indicating that such grafting is possible, at least for small oligomers.
\end{abstract}

Keywords: Grafting

\section{Introduction}

Various studies have been carried out to prove the high electrical conductivity in organic conducting polymers [110]. The properties and characteristics of intrinsically conducting polymers make them suitable materials for several applications such as light-emitting diodes, batteries, gas sensors and activators. Some extensive results on the gas-sensing properties of conducting polyaniline (PAn) and polypyrrole (PPy) have been reported [11-14]. In order to improve mechanical properties of conducting polymers, blending or making composites with other polymers has been proposed. Electrochemically prepared blends of polypyrrole-polyamide (PPy-PA) composite films have better properties for applications like chemical sensors, but their conformational behaviour and electronic properties are still somewhat ambiguous.

In our earlier studies, we have demonstrated that polybisphenol A carbonate (PC) is quite suitable as a host matrix polymer for PPy [8] and PAn [9] in composite synthesis. This was attributed to the low percolation threshold of the two polymers arising from a possible hydrogen-bonding and/ or grafting. Further proof for the above argument was given in an article that offers a significant contribution to the understanding of the possible interactions of PAn with various matrix materials such as PC and PA [15]. PAn-PC compos-

\footnotetext{
* Corresponding author.
}

ites are found to be promising in the detection of ammonia gas $[13,14]$. This behaviour was attributed to the grafting of two polymers leading to a greater surface area, since numerous differences were reported between the as-synthesized films and the simple mechanical mixtures of two polymers (namely, PPy-PC, PAn-PC and PPy-PA). The above argument has been proven to a certain extent via $X$-ray electron spectroscopy (XPS) studies [16].

Having such interesting experimental results led us to investigate the grafting capability of PAn and PPy on insulating polymers such as PC, poly (tetramethyl bisphenol A carbonate) (TMPC) and PA from a theoretical point of view with a special focus on the PPy-PA couple.

\section{Calculations and discussion}

In this study, we employed a semi-empirical quantum mechanical approach utilizing AM1 parameters [17]. Since actual calculations of reasonably long polymer chains still seem to be computationally prohibitive, we have selected some model compounds to study the possibilities of grafting between different polymers. To represent parts of conducting polymers we select dimers of PAn (both in benzoid and quinoid forms) and PPy. The insulating polymers are modelled by ethylamide and bisphenol A carbonate. In Table 1, we present the heats of formation of these compounds obtained by full optimization of the geometric structures. The 
Table 1

The heats of formation $\Delta H_{\mathrm{f}}^{\circ}$

\begin{tabular}{lc}
\hline Molecule & Heat of formation $(\mathrm{kcal} / \mathrm{mol})$ \\
\hline $\operatorname{PAn}(\mathrm{bb})^{\mathrm{a}}$ & 54.62 \\
$\operatorname{PAn}(\mathrm{ab})$ & 89.21 \\
$\operatorname{PPy}(\alpha \alpha)^{\mathrm{b}}$ & 80.61 \\
$\operatorname{PPy}(\alpha \beta)$ & 80.31 \\
$\operatorname{PPy}(\beta \alpha)$ & 80.89 \\
$\operatorname{PPy}(\beta \beta)$ & 80.87 \\
$\operatorname{PA}$ & -36.65 \\
$\operatorname{PC}$ & -84.51 \\
\hline $\mathrm{TMPC}$ & -112.24 \\
\hline
\end{tabular}

${ }^{2} b$ and $q$ denote benzoid and quinoid structures, respectively.

${ }^{b} \alpha$ and $\beta$ denote position where bonding occurs.

benzoid form of the PAn dimer is more stable in the gas phase than the quinoid-benzoid form, as previously reported $[15,18,19]$. Pyrrole dimers can be obtained by various linkages between $\alpha$ and $\beta$ carbons. Even though these structures are of similar stability, they are included in our investigations as they may display different characteristics towards grafting.

To figure out the possible existence of an exotic molecule brings out quite a challenging problem for theoreticians. Under normal conditions, the quantum chemistry offers a strong tool for analysing bonding in relatively small molecules. Even though only the gas phase structures can be studied correctly, one can carry out a large number of 'numerical experiments' to understand the stability and electronic properties where actual experiments may be too difficult or costly. However, these electronic calculations suffer from the fact that only the final products can be studied, that is, to get the reaction mechanisms under real conditions is extremely difficult. One can create a 'theoretical' molecule while, in reality, preparation of such a molecule may not be possible. Then a straightforward application of a geometry optimization algorithm does not suffice and one has to combine various bits of information to find clues. In our problem, we face a similar situation: to understand whether the bonding (grafting) between conducting and insulating polymers can exist, we have to generate such combined molecules and calculate relative stabilities, even though these stabilities do not relate to the activation energy barriers for the formation of such inter-polymer bonding.

In Table 2, we present heats of formation of PAn-PC, PAn-TMPC, PPy-PA, PPy-PC and PPy-TMPC pairs which are depicted in Fig. 1. The stabilities of these theoretically generated molecules do not seem to be so out of place to exclude the existence of such bonding. In addition to the stability measure, we studied the strengths of the bonds formed between two model molecules. The bond orders are all in the range $0.85-0.95$ which clearly signals relatively strong single bonds.

More reliable evidence for the existence of grafting between these molecules could be obtained if the reaction mechanisms for the formation could be established. That is, one needs to find a reasonable reaction coordinate over which the reactants can form a stable product. This process may involve breaking and forming of more than one bond. The problems of establishing the proper reaction coordinate and the observation of the correct reaction mechanism arise purely from numerical aspects of the computational procedures. At each molecular geometry, the optimum structure is obtained either by constrained or unconstrained optimization of the electronic structure with respect to coordinates of atoms. This is an optimization problem in the space of a few hundred dimensions; consequently, the number of local minima is extremely high and, unless one has additional clues to the structure (in that case there may not be any need for a theoretical approach), to find the proper reaction channel is

Table 2

Enthalpies of formation and bond orders for the intermolecular bonds $(\mathrm{N}-\mathrm{C})$

\begin{tabular}{|c|c|c|}
\hline Couple & Heat of formation & Bond order \\
\hline \multicolumn{3}{|l|}{$P A n-P C$} \\
\hline PAn (bb)-PC & -7.35 & 0.93 \\
\hline $\operatorname{PAn}(q b)-P C$ & 28.69 & 0.94 \\
\hline $\operatorname{PAn}(b q)-P C$ & 56.11 & 0.92 \\
\hline \multicolumn{3}{|l|}{$P A n-T M P C$} \\
\hline PAn(bb)-TMPC & -43.89 & 0.93 \\
\hline $\operatorname{PAn}(q b)-T M P C$ & 41.14 & 0.94 \\
\hline $\operatorname{PAn}(b q)-T M P C$ & 38.71 & 0.93 \\
\hline \multicolumn{3}{|l|}{$P P y-P A$} \\
\hline $\operatorname{PPy}(\alpha \alpha)-\operatorname{PA}$ & 43.56 & 0.81 \\
\hline $\operatorname{PPy}(\alpha \beta)-P A$ & 47.74 & 0.83 \\
\hline $\operatorname{PPy}(\beta \alpha)-P A$ & 43.18 & 0.87 \\
\hline $\operatorname{PPy}(\beta \beta)-P A$ & 43.4 & 0.88 \\
\hline \multicolumn{3}{|l|}{$P P y-P C$} \\
\hline $\operatorname{PPy}(\alpha \alpha)-\operatorname{PC}$ & 17.1 & 0.86 \\
\hline $\operatorname{PPy}(\alpha \beta)-P C$ & 13.46 & 0.85 \\
\hline$P P y(\beta \alpha)-P C$ & 12.2 & 0.85 \\
\hline $\operatorname{PPy}(\beta \beta)-\mathrm{PC}$ & 12.09 & 0.85 \\
\hline \multicolumn{3}{|l|}{$P P Y-T M P C$} \\
\hline $\operatorname{PPy}(\alpha \alpha)-\mathrm{TMPC}$ & -6.54 & 0.87 \\
\hline $\operatorname{PPy}(\alpha \beta)-T M P C$ & -11.4 & 0.84 \\
\hline $\operatorname{PPy}(\beta \alpha)-T M P C$ & -12.55 & 0.86 \\
\hline $\operatorname{PPy}(\beta \beta)-T M P C$ & -12.38 & 0.87 \\
\hline
\end{tabular}
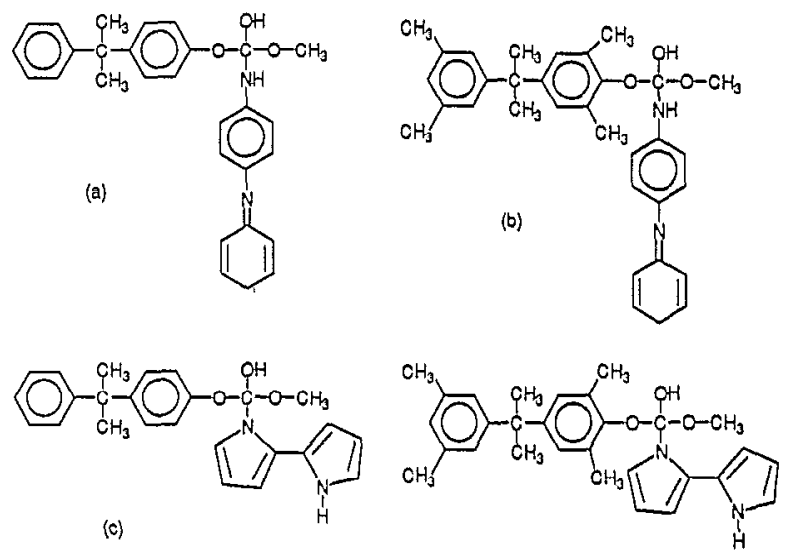

(d)

Fig. 1. The estimated model of the couples: (a) PAn-PC; (b) PAn-TMPC; (c) PPy-PC; and (d) PPy-TMPC. 
a serious challenge. On the other hand, even if the computations do not reveal such a channel, one cannot exclude the possibility of the reaction.

In the light of all these difficulties, we have tried various reaction coordinates which may seem to be reasonable approximations to the grafting processes. Our first model reaction occurs between ethylamide and $\alpha \alpha$-bipyrrole. When these two molecules come within the interaction range, there should be a hydrogen shift from the nitrogen of the pyrrole ring to the oxygen of the amide, and a bond forms between the nitrogen and the carbon of ethylamide. From a collision theory point of view, this shift occurs only when two molecules collide on a minimum energy path and if the energies are in the correct range. Since the majority of the quantum chemical calculations are within the time-independent mode (a study of quantum dynamics is still not possible for a molecule of this size), it is not easy to observe the hydrogen shift. However we were able to detect the possibility of such a mechanism, though somewhat indirectly. Our standard reaction mechanism calculations (bringing two molecules together from a large separation) based on the minimum energy reaction coordinate produced a repulsive curve with no detectable minimum. Then we tried a different approach to check whether the repulsive behaviour of the potential curve represented the actual interaction or a computational artifact, as we were not able to locate the global minima. We started from an initial configuration of two very closely lying molecules (hydrogen atom is still attached to the nitrogen of the pyrrole ring) and let it relax. In this manner we were able to generate conditions of a highly energetic collision.

In Fig. 2, we present both curves and this new potential function which corresponds to the relaxation process clearly displays a minimum. In addition to these reaction mechanisms, we have searched various starting configurations to look for different mechanisms and another reaction possibility is detected which results in elimination of an ammonia molecule although ethylamide is now connected to a $\beta$ carbon. These three processes are denoted as (a) slow approach of bipyrrole and ethylamide (repulsive), (b) relaxation from

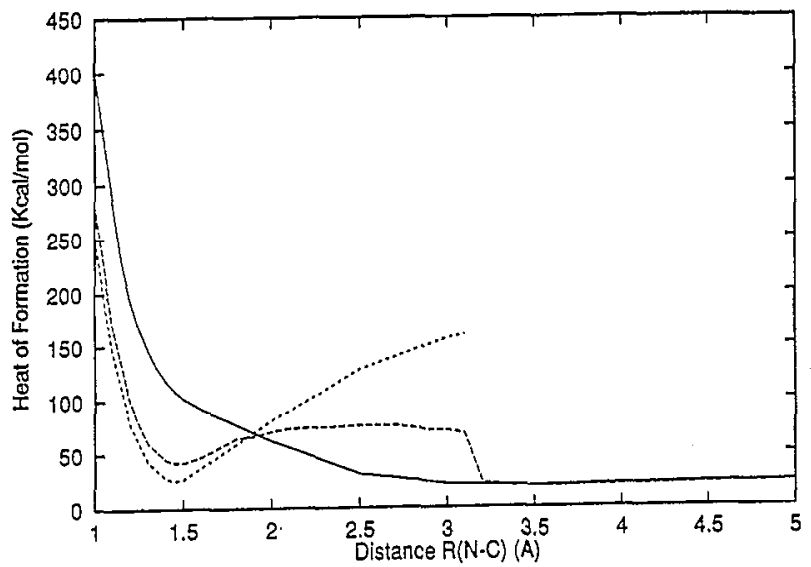

Fig. 2. Potential energy curves for the interaction between pyrrole dimer and ethyl amide: ——, repulsive approach; - - - $\longrightarrow$, relaxation; - - -, ammonia-producing process. an artificially formed pair at very short intermolecular distance and (c) ammonia-releasing process. The detailed anal$y$ sis of the electronic structures at three intermolecular distances $(R(\mathrm{~N}-\mathrm{C})=5 \AA$ for separated molecules, $R=1.5$ $\AA$ for the minimum energy structure and around $R=2.0 \AA$ for the crossing point of different potential energy functions) can be summarized as follows.

Fig. 3(a) depicts the structure of the minimum energy conformation which is obtained from the relaxation path in which the hydrogen shift from the nitrogen on the pyrrole ring to the oxygen of the carbonyl group occurs around $R=1.00 \AA$. The $\mathrm{N}-\mathrm{C}$ bond length is $R=1.50 \AA$, the bond order is 0.882 and the total charge on the bipyrrole part of the molecule is around -0.18 a.u. Bond strengths of the pyrrole rings as well as the pyrrole-pyrrole bond do not show any significant difference to those of the unsubstituted bipyrrole. The bond between the oxygen and the shifted hydrogen has a length of 0.964 and order of 0.927 , which point to a stable bonding between bipyrrole and cthylamide. The minimum energy conformation for the ammonia-releasing process (Fig. 3(b)) corresponds to 2-(2-pyrolyl),3propionylpyrrole, which actually is a lower energy conformation. However, at the moment we do not have any experimental evidence to the possibility of such a reaction and we do not pursue this process any further.
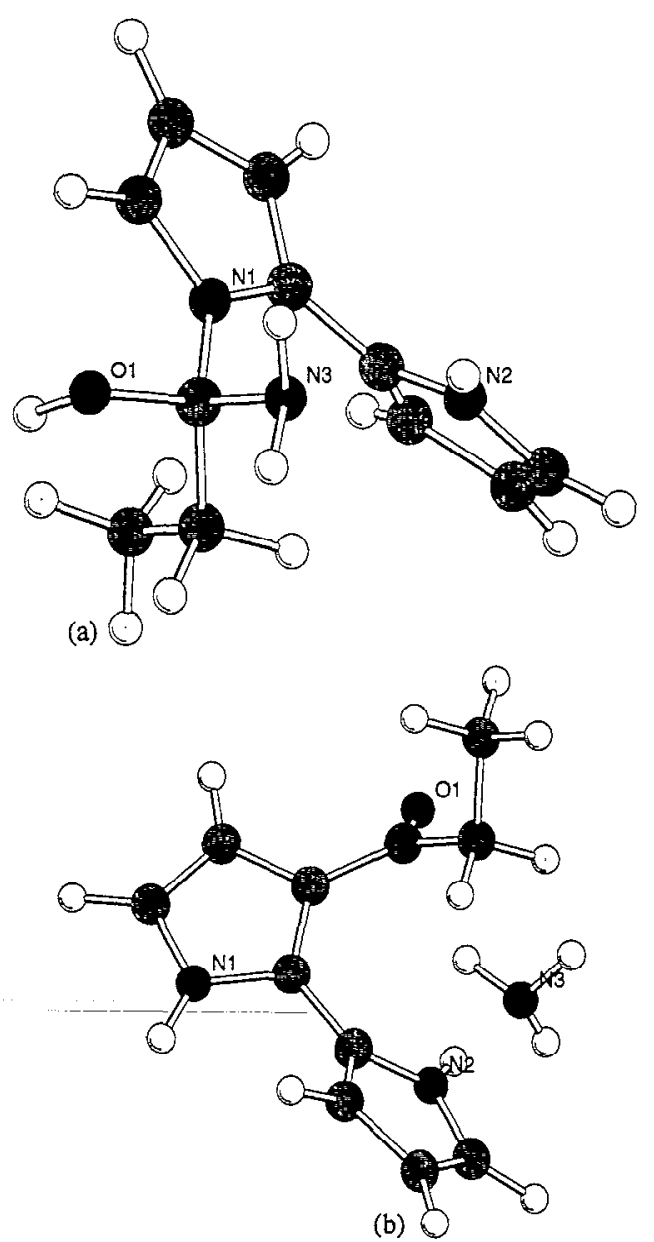

Fig. 3. (a) Hydrogen-shifted grafting pair of ethyl amide and $\alpha \alpha$-bipyrrole. (b) Conformation for the ammonia-2-(2-pyrolyl),3-propionylpyrrole pair. 
The crossing point is $2.0 \AA$ for two potential curves and, even though the relaxation curve is above the repulsive one, we note that the hydrogen shift is reversed around $3.2 \AA$ and we observe the correct asymptotic behaviour.

The calculations on other couples of insulating and conducting polymers fail to display such different characteristics or energy minima. This lack of evidence cannot be interpreted as proof of the nonexistence of the grafting. Work along these lines is still in progress.

\section{Conclusions}

We have studied the interaction mechanisms of the bipyrrole-ethylamide couple with quantum mechanical procedures. There are three different interaction pictures which can be classified as totally repulsive, two metastable states corresponding to different channels. In the light of the difficulties of the self-consistent-field method and numerical optimization procedures, we believe that all three reaction mechanisms are likely to occur, especially for polymeric systems where the intermonomer distances between different chains can easily fall into the attractive regimes. Even though the observed energy minimum is above the energy of two separate molecules, the height of the activation energy barrier (around 34 $\mathrm{kcal} / \mathrm{mol}$ ) is sufficient to trap a number of couples so that grafting is possible. The experimental evidence point to a very small amount of grafting, and the theoretical calculations exactly parallel these findings, that is, the bonding between pyrrole and ethylamide is likely to occur under convenient conditions.

\section{Acknowledgements}

This process is partially supported by the Volkswagen Foundation, Grant I/69 797.

\section{References}

[1] J.P. Pouget, M. Laridjani, M.E. Jozefowicz, A.J. Epstein, E.M. Scherr and A.G. MacDiarmid, Synth. Met., 5 I (1992) 95.

[2] S. Stafström, J.L. Brédas, A.J. Epstein, W.S. Huang and A.G. MacDiarmid, Phys. Rev. Lett., 59 (1987) 1464.

[3] J.L. Brédas, C. Quattrocchi, J. Libert, A.G. MacDiarmid, J.M. Ginder and A.J. Epstein, Phys, Rev. B, 44 (1991) 6002.

[4] M. De Paoli, R.J. Waltmann, A.F. Diaz and J. Bargon, J. Polym. Sci, Polym. Chem., 23 (1985) 1687.

[5] O. Niwa, M. Kakuchi and T. Tamamura, Macromolecules, 20 (1987) 749.

[6] X. Bi and Q. Pei, Synth. Met., 41-43 (1987) 145.

[7] B. Zinger and D. Kijel, Synth. Met. 4I-43 (1991) 1013.

[8] H.L. Wang, L. Toppare and J.E. Fernandez, Macromolecules, 23 (1990) 1053.

[9] S. Doğan, U. Akbulut and L. Toppare, Symth. Met. 53 (1992) 29.

[10] F. Selampinar, U. Akbulut, T. Yalçin, Ş. Süzer and L. Toppare, Synth. Met., 62 (1994) 201.

[11] P.N. Bartlett, P.B.M. Archer and S.K. Ling-Chung, Sensors and Actuators, 19 (1989) 125.

[12] H.V. Shurmer, P. Corcoran and J.W. Garner, Sensors and Actuators $B, 4$ (1991) 29 .

[13] S. Doğan, U. Akbulut, T. Yalçin, Ş. Süzer and L. Toppare, Synth. Met., 62 (1993) 27.

[14] F. Selampinar, L. Toppare, U. Akbulut, T. Yalçin and Ş. Süzer, Synth. Met., 68 (1994) 109.

[15] S. Bahçeci, L. Toppare and E. Yurtsever, Synth. Met., 68 (1994) 57.

[16] S.. Süzer, L. Toppare, G.C. Allen and K.R. Hallam, J. Mol. Struct., (1994) in press.

[17] MOPAC version 6.00, QCPE No. 455.

[18] S. Stafström and J.L. Brédas, Synth. Met., 14 (1986) 297.

[19] D.S. Boudreaux, R.R. Chance, J.F. Wolf, L.W. Shacklette, J.L. Brédas, B. Thémans, J.M. André and R. Silbey, J. Chem. Phys, 85 (1986) 4584. 